\title{
Investigation of hydraulic fitting losses
}

\author{
Marian Bojko ${ }^{1, *}$, and Milada Kozubková ${ }^{1}$ \\ ${ }^{1}$ Department of Hydromechanics and Hydraulic Equipment, Faculty of Mechanical Engineering, \\ VŠB-Technical University of Ostrava, 17. listopadu 15, 70833 Ostrava - Poruba, Czech Republic
}

\begin{abstract}
Fluid flow in hydraulic systems, fittings, and piping causes hydraulic losses due to the change of flow direction and friction in the fluid. The main consequence of the resulting losses is the increase in the overall pressure gradient of the circuit. The paper is focused on the investigation of valve losses and the determination of resistance coefficients, the definition of which depends on the valve type. Methods of determination are of two types, i.e. experimental methods and mathematical methods. In the case of experimental methods, the procedures prescribed by the standards and regulations must be respected. Mathematical methods are related to defining an appropriate mathematical model and numerical approach. Both methods are applied to the ball valve and model verification is performed at the same time. The output of the thesis is the methodology for determination of resistance coefficients of valves with other diameters and other flowing media based on verified mathematical modeling.
\end{abstract}

\section{Introduction}

When fluid flows through the hydraulic circuint, there are various hydraulic losses due to the viscosity of the fluid and the change of direction of flow. As a result, it is necessary to select a pump with appropriate performance parameters. This consequence must not be neglected in the design and calculation of the circuit, so that the sum of all the pressure losses in the circuit will be greater than the pressure supplied by the pump. The secondary adverse effect is the transformation of the hydraulic loss energy into heat and hence the heating of the circuit elements and the fluid, which is associated with other demands on the choice of appropriate cooler. The resulting hydraulic resistances are defined by various types of loss coefficients that are specific to the individual circuit elements $[1,2]$.

-local loss coefficient $\zeta$ is determined when fluid is flowing in areas where the flow direction of the medium (knees, arcs, branches, compensating loops, etc.) changes,

-coefficient of friction losses $\lambda$ is typical coefficient for the fluid flow in pipelines,

-flow coefficient $\mu$ is typical in the areas where the flow is throttled (check valves, pressure reducing valves, ball valves, etc.).

\footnotetext{
*Corresponding author: marian.bojko@,vsb.cz
} 
In nowday of the modern computational technology, it is possible to identify hydraulic resistances in addition to the methodology of experimental approach by a methodology based on mathematical approach and using various software (ANSYS Fluent, Matlab, Maple, MathCAD, etc.). It is important to verify this methodology as each solution can be burdened a certain computational error due to the mathematical model used. When modeling the flow in hydraulic systems, it is necessary to know the characteristics and resistance coefficients of the individual elements and, if necessary, to adapt them to a particular variant of the mathematical model. The admission of mathematical modeling into the technical sector has brought a number of advantages, for example, to find out all the information about the behavior of the whole mechanism, to perform simulation of flows in elements where physical experiments appear to be very difficult, to simplify the design the entire hydraulic system $[3,4]$.

\section{Design, measurement of characteristics, specific valve parameters}

Hydraulic circuits include valves of various designs and designs. Their distribution is according to the type and construction of the control element, which may be a ball, a bow, a plate, a saddle and the like. Then the valves according to the design can perform various functions in the hydraulic circuits, especially control and flow control. The most common types include ball valves, bbutterfly valves, gate valves, globe valves and others, see Figure 1.
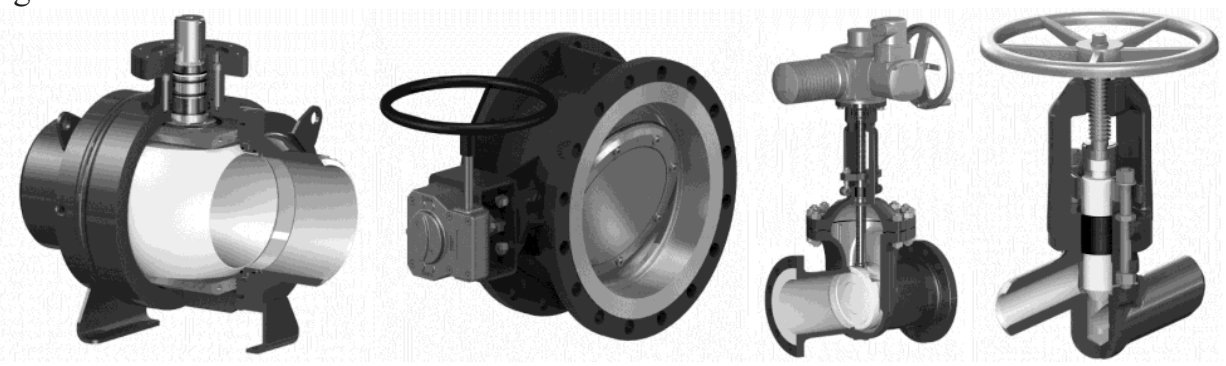

Fig. 1. Ball valves, Butterfly valves, gate valves, globe valves [5].

The evaluation of the characteristic of the valves and the respective loss factors (loss coefficient $\zeta$, flow coefficient $\mu$, effective surface $\mathrm{S}$ ) and other parameters can be realized by experimental measurements on the hydraulic circuit according to the requirements of the standard. The results of experimental measurements of different types of valves are presented in publications $[6,7]$ including the relevant characteristics. The basic characteristic of the valve is the dependence of the pressure drop $\Delta p$ on the flow rate $Q$. This characteristic consists of sub-characteristics, which are determined for selected strokes of the poppet control valve or for selected angles of rotation of the ball in the case of ball valves. Typical characteristics $(\Delta p=f(Q), \zeta=f(z))$ for the poppet valve obtained from the measurements are shown in Figure 2, Figure 3. 


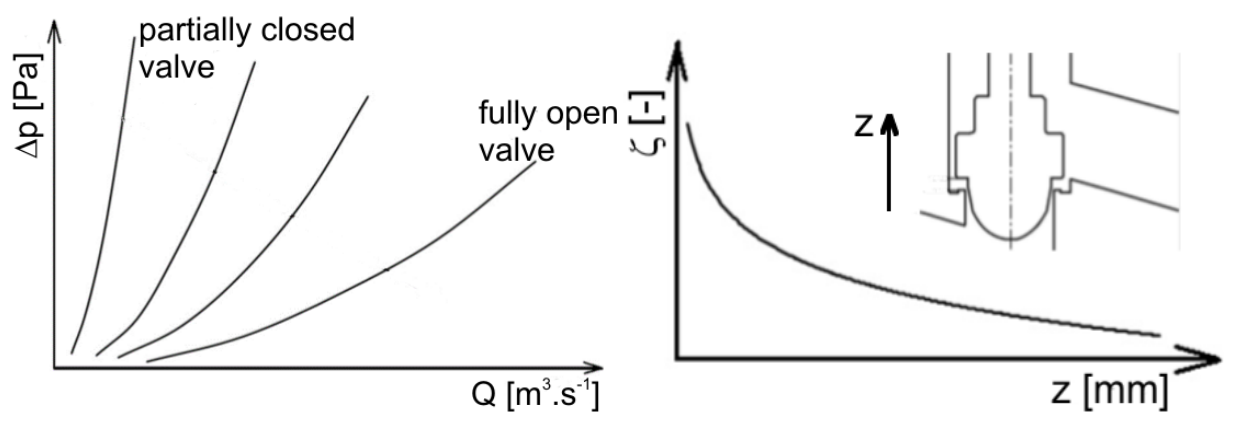

Fig. 2. Valve characteristic.

Fig. 3. Loss coefficient's dependence on the valve stroke.

From graphical plotting of the measured values, it is possible to determine the behavior of the valve at different flow rates and to select a suitable valve for the given application based on this information. When creating of the circuit and the following measurement, the principles given by the standard [8] have to be met so that the loss factors are universally used. Scheme of the measuring system according to ČSN EN 60534-2-3 is shown in Figure 4.

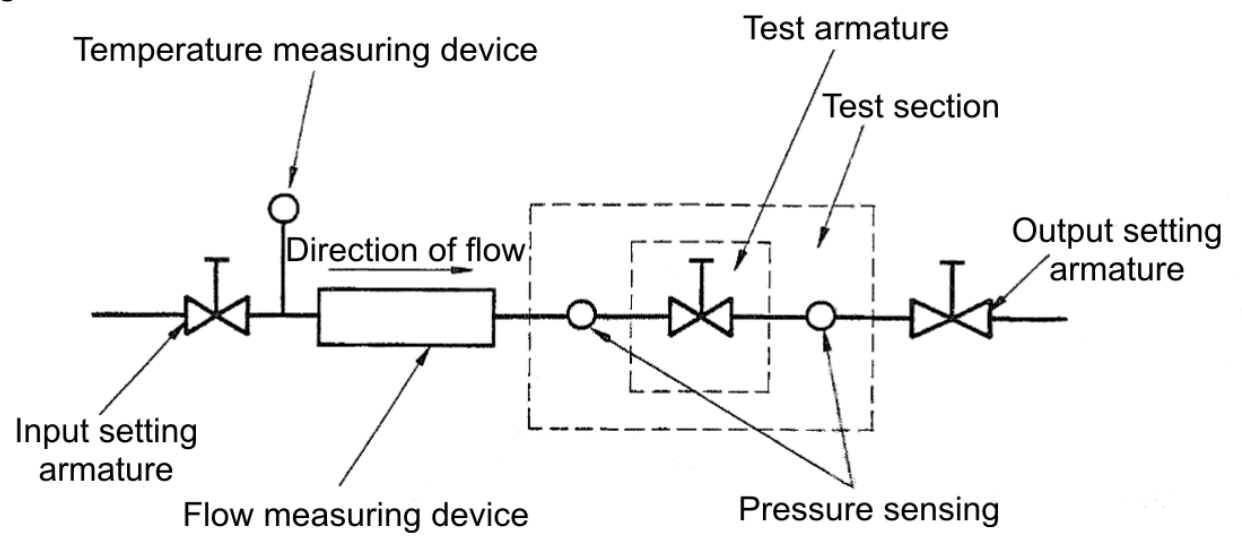

Fig. 4. Diagram of flow test system.

A second approach to investigating the characteristics of valves of different constructions and types is mathematical flow modeling or CFD analysis. CFD analysis can be performed on the 3D models of individual valves and the application of suitable turbulent models to obtain the relevant characteristics. Then, it can be verified by experimental measurements. Studies on mathematical modeling are presented in papers [9, $10,11,12]$.

\subsection{Basic valve parameters}

The basic parameters of the valve are nominal diameter DN, nominal pressure PN, maximum working temperature of the valve and medium, loss $\zeta$ and discharge coefficient $\mu$, effective area $S$ and others, including the cavitation coefficient.

The loss coefficient is derived from the Bernoulli equation and $\Delta p=f(Q)$ characteristic.

$$
\Delta p=\rho \zeta \frac{u^{2}}{2}=\rho \zeta \frac{Q^{2}}{2 S^{2}}
$$


For the measured sub-characteristic, the loss coefficient is determined by the least squares statistical method. In the case of a flow through a narrowed area (valve, diaphragm, nozzle, etc.) the inverse relation to equation (1) is used more frequently

$$
Q=\mu S \sqrt{\frac{2}{\rho}} \cdot \sqrt{\Delta p}
$$

Comparing both equations $\frac{\Delta p}{Q^{2}}=\frac{\rho}{2} \frac{\zeta}{S^{2}}$ a $\frac{\sqrt{\Delta p}}{Q}=\sqrt{\frac{\rho}{2}} \cdot \frac{1}{\mu S} \Rightarrow \frac{\Delta p}{Q^{2}}=\frac{\rho}{2} \frac{1}{\mu^{2} S^{2}}$ the following dependence between the loss and discharge coefficient can be derived

$$
\mu=\frac{1}{\sqrt{\zeta}}
$$

For hydraulic element with variable flow area (using Matlab-Simulink-SimHydraulics software) instead of the loss or discharge coefficient, the effective area depending on the position and, respectively opening is used. Variable area of the orifice is not identical to the geometric area because it is modified by contraction and swirling when the fluid flows around the edges of the orifice. If the valve is completely open, then the orifice area is maximum area and is equal to the flow area of the connected tubes $S_{0}$. Discharge coefficient can then be determined from the loss coefficient $\zeta$ obtained from the $\Delta p=f(Q)$ sub-characteristic for the full opening of the valve and will be constant even for the subcharacteristics when opening the valve (eg $20^{\circ}, 40^{\circ}$ and $60^{\circ}$ ). For other opening therefore, the area of the orifice, which is determined from the experimentally measured characteristic (discharge coefficients), must be changed. The following effective area for partial closing of the valve eg $20^{\circ}, 40^{\circ}$ and $60^{\circ}$ is determined from the other measured sub-characteristics $\Delta p=f(Q)$, but the discharge coefficient $\mu$ will be constant $\left(\mu_{0}=\mu\right)$. Thus, for a partial closing of the valve, for example by $20^{\circ}$, the effective area is determined:

$$
S_{20}=\frac{\mu_{20}}{\mu} \cdot S_{0}
$$

Further effective areas of the orifice are determined analogously.

Depending on the standard or use in different countries with different physical unit systems, other variants of the discharge coefficient labeled as $\mathrm{A}_{v}, \mathrm{~K}_{\mathrm{v}}$ a $\mathrm{C}_{\mathrm{v}}$ are used.

\section{Definition of mathematical model of flow through the ball valve}

The defined mathematical model characterizes the flow rate of water in the general spatial geometry presenting the inner space of the ball valve at different closure positions. The general model specification is as follows:

- 3D flow of one phase (water).

- Flow of incompressible liquid, $\rho=$ const.

- Steady flow, $\frac{\partial}{\partial t}=0$.

- Isothermal flow, $T=$ const.

- Turbulent flow.

In the case of turbulent flow, and due to swirling when the fluid flows around the edges of the ball valve orifice, it appears to be an optimal the two-equation $k-\varepsilon$ RNG mathematical model, which consists of the following basic system of balance equations in vector notation [3]: 
continuity equation

$$
\frac{\partial \rho}{\partial t}+\nabla \cdot(\rho \vec{u})=S_{z}
$$

Navier-Stokes equation

$$
\frac{\partial(\rho \overrightarrow{\bar{u}})}{\partial t}+\nabla \cdot(\rho \overrightarrow{\bar{u}} \vec{u})=-\nabla \bar{p}+\rho \vec{a}+\nabla \cdot\left(\begin{array}{l}
= \\
\bar{\tau}_{t}
\end{array}\right)+S_{m}
$$

Equation for turbulent kinetic energy $k$

$$
\frac{\partial}{\partial t}(\rho k)+\nabla \cdot(\vec{u} k)=\nabla \cdot\left(\frac{v_{t}}{\sigma_{k}} \nabla k\right)+\rho P-\rho \varepsilon
$$

Equation for tubulent dissipation rate

$$
\frac{\partial}{\partial t}(\rho \varepsilon)+\nabla \cdot(\vec{u} \varepsilon)=\nabla \cdot\left(\frac{v_{t}}{\sigma_{\varepsilon}} \nabla \varepsilon\right)+C_{1 s} \rho P-C_{2 s} \rho \frac{\varepsilon^{2}}{k}
$$

and Boussineq's analogy is used to calculate Reynolds' strains.

The RNG k- $\varepsilon$ model as a modification of the Standard k-e turbulent model using the renormalization group method RNG [3] was chosen and was solved by final volume numerical method. At the input of the computation area, the VELOCITY INLET conditions were defined and at the outlet the PRESSURE OUTLET were used. The flowing substance was defined as water with corresponding physical properties (density, kinematic viscosity).

\section{Results of the experimental part and mathematical modeling}

In the experimental part were measured $\Delta p=f(Q)$ of the ball valve characteristic for each valve position $\left(0^{\circ}, 20^{\circ}, 40^{\circ}, 60^{\circ}\right)$. Ball valves are hydraulic elements in the circuit that are intended to close of the flow rate through piping with help of rotating the ball with the hole (see Figure 1). Depending on its opening or closing, the flow rate is controlled, but the valves can not be used as throttle or regulating valves. This valve produces a minimum pressure drop when fully opened. The range of use of valve depends on the type and temperature of the flowing medium and material. Measurement of the static characteristics of the ball valve was performed in a laboratory on a water line of plastic components. The ball valve in the circuit has established sampling points before and after the element according to EN 60534-2-3, which are connected to an inverted U-tube for measuring differential pressure. The flow rate through the circuit is measured by the clone (crosssectional gauge) through the loss height on the U-tube of the clone and the given calibration curve of the clone. 


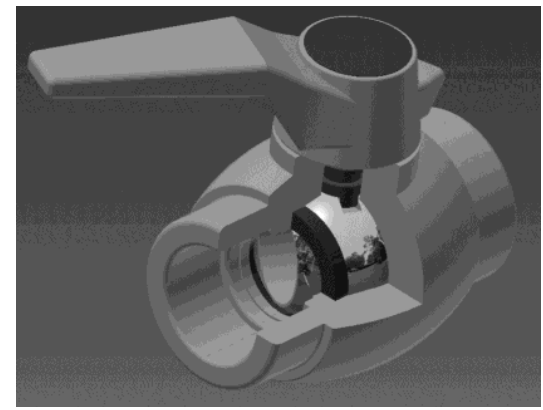

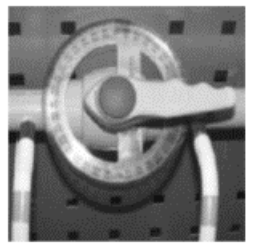

$\alpha=0^{\circ}$

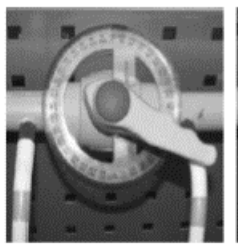

$\alpha=20^{\circ}$

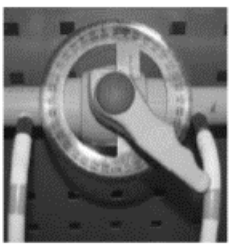

$\alpha=40^{\circ}$

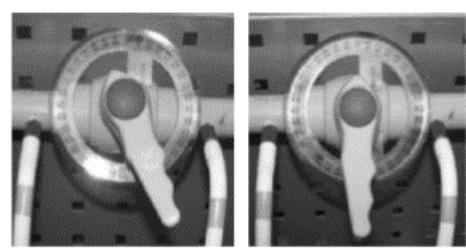

$\alpha=60^{\circ}$

$\alpha=90^{\circ}$

Fig. 5. Ball valve model, valve postions.

\subsection{Evaluation of experimental measurements}

Based on measured and calculated values, it is possible to plot $\Delta p=f(Q)$ characteristics of the ball valve for different angles of closure see Figure 6. From the distribution, there is a visible difference between the individual characteristics for the individual $\left(0^{\circ}, 20^{\circ}, 40^{\circ}, 60^{\circ}\right)$.

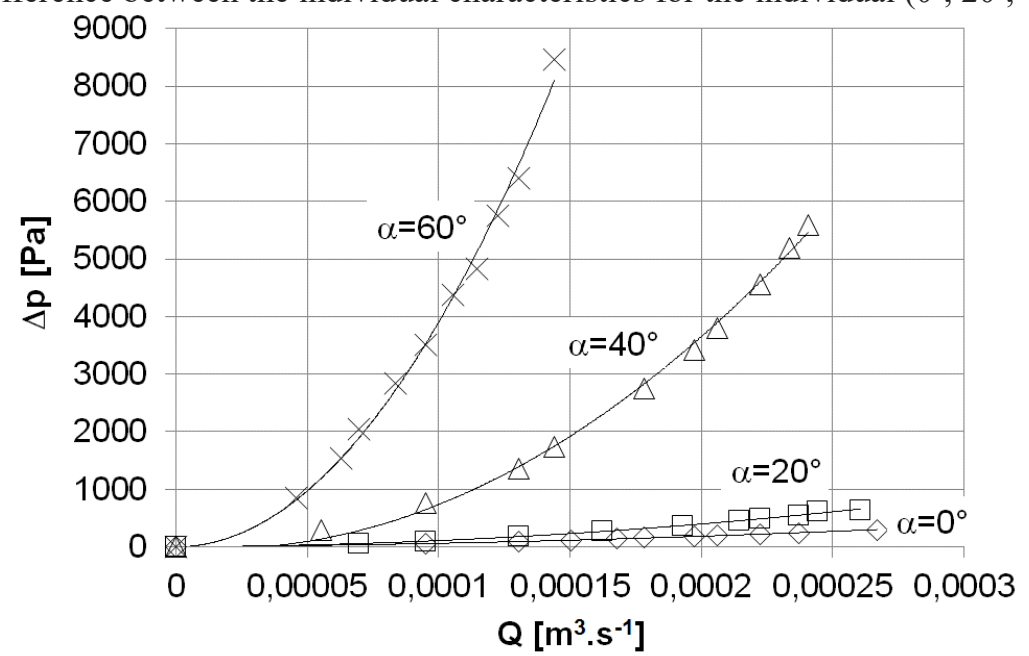

Fig. 6. Experimentally measured characteristic $\Delta p=f(Q)$ ball valve for individual closures $\left(0^{\circ}, 20^{\circ}\right.$, $\left.40^{\circ}, 60^{\circ}\right)$.

Then from the individual $\Delta p=f(Q)$ characteristic were calculated by the least squares method, the loss coefficients $\zeta$, the flow coefficients $\mu$ and the effective area $S$, which will then be compared with the CFD analysis of the flow. 


\subsection{Evaluation of mathematical modeling}

\subsubsection{Model ball valve, computational grid}

For the CFD analysis of the ball valve flow, a model corresponding to the internal volume of flowing water was created for the subsequent creation of the computational grid. A 3D design model of the ball valve from Inventor (Figure 5) was used to create the final model. This model was subsequently opened in ANSYS DesignModeler in the appropriate format for CFD analysis requirements. In this way four models (Figure 7) corresponding to the fully open valve, the valve in the open position $20^{\circ}, 40^{\circ}$ and $60^{\circ}$ were created [13]. Then, a corresponding computational grid was generated in these models. Software ANSYS Meshing was used to create the computational grid. In the region of the valve itself and in particular in the area of closure, a finer grid was created than in the areas of valve connection to the pipeline.
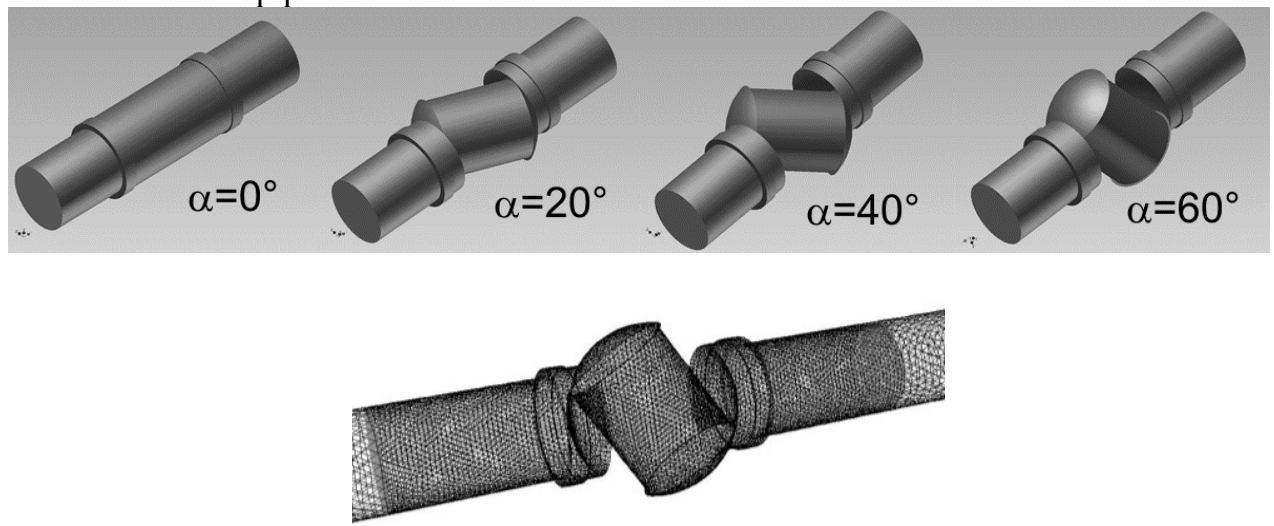

Fig. 7. Resulting models in position $0^{\circ}, 20^{\circ}, 40^{\circ}$ a $60^{\circ}$, computational grid for the model in position $60^{\circ}[13]$.

Then the basic current variables such as pressure and velocity in individual cuts were evaluated in detail to map the flow characteristics of each ball valve position. The longitudinal section pressure field for the valve closing position of $20^{\circ}$ is shown on Figure 8.

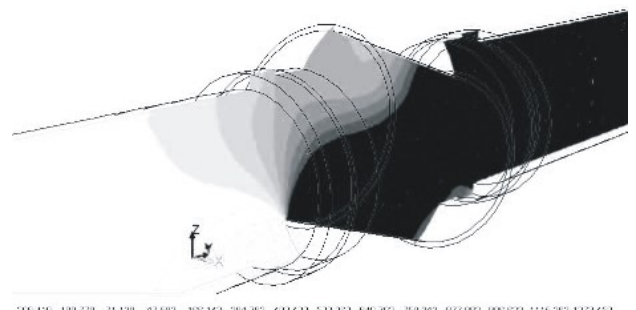

Fig. 8. pressure field $(-300 \div 1300)$ Pa for closed position $20^{\circ}$.

In the next phase the $\Delta p=f(Q)$ characteristics from the mathematical modeling of the flow for the different valve closing positions are evaluated. Then loss factors $\zeta$, flow coefficients $\mu$ and specific area $S$ are calculated, which will then be compared with experimental measurement. 


\section{Comparison of experimental measurement and mathematical modeling}

Experimental measurements and mathematical modeling were performed under the same boundary conditions. The resulting experimentally measured $\Delta p=f(Q)$ ball valve characteristic for the different closing positions is shown in Figure 5. Subsequent comparison of the experimental measurement with the results of the mathematical modeling is focused on the evaluation of the loss coefficient $\zeta$ the flow coefficient $\mu$ and the reference area $S$ using the experimental measurement and CFD analysis. The resulting values for the individual closing positions are shown in Table 1.

Table 1. Evaluation $\zeta, \mu, S$ based on experimental measurement and mathematical modeling.

\begin{tabular}{|c|c|c|c|c|}
\hline \multirow{2}{*}{} & \multicolumn{4}{|c|}{ Experimental measurements } \\
\cline { 2 - 5 } & \multicolumn{4}{|c|}{ Closure angle } \\
\cline { 2 - 5 } & $0^{\circ}$ & $20^{\circ}$ & $40^{\circ}$ & $60^{\circ}$ \\
\hline Loss coefficient $\zeta[1]$ & 0.579 & 1.271 & 11.928 & 50.545 \\
\hline Flow coefficient $\mu[1]$ & 1.314 & 0.887 & 0.290 & 0.141 \\
\hline Efective a $S\left[\mathrm{~mm}^{2}\right]$ & 254.469 & 171.699 & 56.055 & 27.231 \\
\hline & & \multicolumn{4}{|c|}{ Mathematical modeling } \\
\hline Loss coefficient $\zeta[1]$ & 0.672 & 1.450 & 12.813 & 52.124 \\
\hline Flow coefficient $\mu[1]$ & 1.220 & 0.830 & 0.279 & 0.139 \\
\hline Efective a $S\left[\mathrm{~mm}^{2}\right]$ & 0.672 & 1.450 & 12.813 & 52.124 \\
\hline
\end{tabular}

Further, the loss coefficient $\alpha$ and the effective area $S$ for the individual closure positions are plotted by graphical dependencies $\zeta=f(\alpha)$ a $S=f(\alpha)$, Figure 9 .

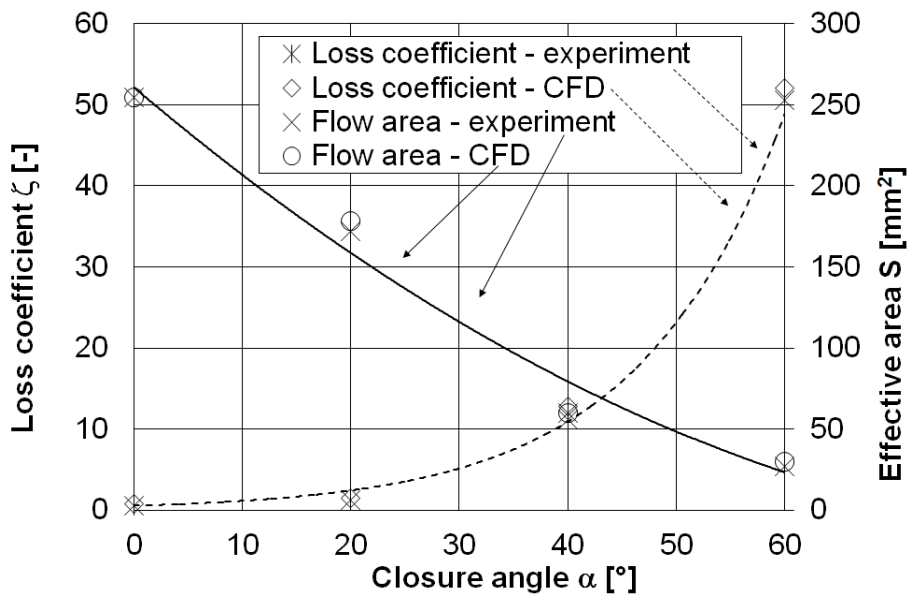

Fig. 9. Dependency of the loss factor $\zeta$. 
The difference between the experimental and mathematically determined values of the resistance factors was up to $15 \%$. The maximum value was reached for maximum opening because the mathematical model did not include the transition between the pipe and the valve. This transition is determined by the solid welding of plastic elements and therefore its geometry is undefined. In the case of partial closure of the valve, the effect of this local loss is negligible compared to local loss due to flou around the edges of the valve hole.

\section{Conclusion}

The aim of the paper was to create an experimental device and a mathematical model to determine the characteristics of the ball valve. In the introductory part the theory of characteristics and resistance coefficients of different types of valves is treated in detail. Measurement of characteristics for the selected ball valve and mathematical modeling under the same boundary conditions was performed. The optimal turbulent mathematical model was chosen by the testing, which took into account the problematic swirl flow at partial opening of the valve. The results of mathematical modeling were compared with experimental measurements in terms of loss coefficient, discharge coefficient and effective area. Analogously, it is possible to investigate the characteristics of valves of different designs in connection with the evaluation of specific resistance coefficients. Mathematical modeling of resistive coefficients is important in cases of large-diameter fittings and for specific media where experimental testing is problematic.

Work was supported by specific research SP2018/157 (Modelování a experimentální ověření dynamických jevů $\mathrm{v}$ tekutinových a vakuových systémech) and by the European Regional Development Fund in the Research Centre of Advanced Mechatronic Systems project, project number CZ.02.1.01/0.0/0.0/16_019/0000867 within the Operational Programme Research, Development and Education.

\section{References}

1. F.P. Incopera, D.P. Dewitt, T.L. Bergman, A.S. Lavine, Fundamentals of heat and mass transfer. 6th ed., 997 (2006)

2. S. Drábková, a kol. Mechanika tekutin. VŠB-TU Ostrava, 260 (2007)

3. M. Kozubková, Modelování prouděn FLUENT, CFX, VŠB-TU Ostrava, 142 (2008)

4. M. Kozubková, T. Blejchař, M. Bojko, Modelování přenosu tepla, hmoty a hybnosti, VŠB-TU Ostrava, 174 (2011)

5. http://www.armaturygroup.cz/en/valves

6. B. Ulanicki, L. Picinali, T. Janus, Measurements and analysis of cavitation in a pressure reducing valve during operation - a case study. Procedia Engineering, 119, 270-279 (2015)

7. J. Jablonská, M. Kozubková, Flow characteristics of control valve for different strokes. EPJ Web of Conferences, 114, 5 (2016)

8. ČSN EN 60534-2-3 Regulační armatury pro průmyslové procesy - Č́st 2-3: Průtok Zkušební postupy. Prosinec (1999)

9. A.S. Tabrizi, M. Asadi, G. XIE, G. Lorenzini, C. Biserni, Computational FluidDynamics-Based Analysis of a Ball Valve Performance in the Presence of Cavitation. Journal of Engineering Thermophysics. 23, 1, 27-38 (2014) 
10. S.C. Zhang, Y.L. Zhang, Z.M. Fang, Numerical simulation and analysis of ball valve three-dimensional flow based on CFD. 26th IAHR Symposium on Hydraulic Machinery and Systems. 1-5 (2012)

11. A. Azad, D. Baranwal, R. Arya, N. Diwakar, Flow Analysis of Butterfly Valve Using CFD. International Journal Of Modern Engineering Research. 4, 6, 50-56 (2014)

12. V.J. Sonawane, T.J. Rane, A.D. Monde, R.V. Vajarinkar, P.C. Gawade, Design and Analysis of Globe Valve as Control Valve Using CFD Software. IOSR Journal of Mechanical and Civil Engineering (IOSR-JMCE). 63-71 (2014)

13. J. Rak, Matematické modelování proudění v kulovém ventilu. Diplomová práce, (2014) 